\title{
Introduction to the Issue on Nanophotonics
}

$\mathbf{N}$ ANOPHOTONICS is a rapidly growing field that addresses a broad spectrum of optics on the nanometer scale, covering technology and basic science. Compared to the behavior of isolated molecules or bulk materials, the behavior of nanostructures exhibit important physical properties that are not necessarily predictable from the observations of either individual constituents or large ensembles. Applying the concepts of metallic and dielectric nanostructures to the interaction of light and matter on subwavelength scales can lead to progress in a variety of scientific and technological fields. On the technological side, there are topics such as nanolithography, high-density optical data storage, as well as applications in high-bandwidth communications, efficient solar power generation, displays, biotechnology, and medicine. On the basic sciences end, in addition to the research and development in applications areas, there is a long list of topics including atomphoton interactions in the optical near-field, and their potential applications for atom trapping and manipulation experiments, microscopy and imaging with subwavelength resolution, linear and nonlinear spectroscopy of nanostructures, nanocavities and nanoapertures, light in confined structures, photonic bandgap (PBG) structures, quantum dots, and plasmon optics.

In this issue of the IEEE JOURNAL OF SELECTED TOPICS IN QUANTUM ELECTRONICS, we feature papers on the science and technology of nanophotonics. We have 19 invited papers along with seven contributed papers covering a wide range of topics in the latest research areas. Several papers focus on the research on photonic crystals and photonic bandgap structures and applications in integrated optics, beam steering, switching, and optical resonators, as well as nanostructured fibers. Nanoplasmonics utilizing large field enhancement with metallic nanostructures with applications in nanoapertures, subwavelength photonic devices and surface plasmon polariton waveguides are reviewed in detail. More traditional structures in integrated optics utilizing nanofabrication and high-index contrast waveguides are also discussed. Another focus area is photonics metamaterials at optical frequencies and negative index materials. Quantum dots, nanowires, and quantum-well structures for optoelectronic applications in light emitters and photodetectors are reviewed. Two papers are in the emerging new area of nanobiophotonics, discussing its applications in laser nanosurgery and virus detection. The other papers are related to near-field optics, study of Si-based nanocrystals and applications in light amplification, and carbon nanotubes.
We hope you will find this issue on nanophotonics interesting and useful. While we cannot claim a comprehensive coverage of all topics under nanophotonics, we believe that this issue will serve as a valuable reference for the readers.

\section{ACKNOWLEDGMENT}

The Guest Editors are grateful to the authors for meeting the deadlines, and the reviewers who responded graciously and quickly when asked for help and provided thoughtful comments that improved the quality of the papers in this issue. The Guest Editors owe special thanks to Chin Tan-yan for her invaluable assistance and dedication in coordinating the manuscripts and assembling this issue, and Linda Matarazzo for her unwavering support.

S. Ünlü and B. Goldberg acknowledge partial support from the National Science Foundation NIRT program ECS-021075 and AFOSR MURI F-49620-03-1-0379. E. Ozbay acknowledges partial support from EU NoE-PHOREMOST and NoEMETAMORPHOSE projects for the preparation of this issue.

\author{
M. SELIM ÜNLÜ, Guest Editor \\ Department of Electrical and Computer Engineering \\ and Photonics Center \\ Boston University \\ Boston, MA 02215 USA
}

EKMEL OZBAY, Guest Editor

Nanotechnology Research Center

Bilkent University

Ankara 06800, Turkey

BENNETT B. GOLDBERG, Guest Editor

Boston University

Boston, MA 02215 USA

NIEK F. VAN HuLST, Guest Editor

Institute of Photonic Sciences (ICFO)

Barcelona 08860, Spain 


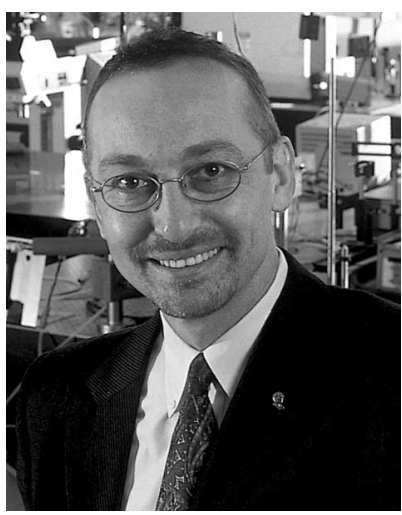

M. Selim Ünlü received the B.S. degree from Middle East Technical University, Ankara, Turkey, in 1986, and the M.S.E.E. and Ph.D. degrees from the University of Illinois, Urbana-Champaign, in 1988 and 1992, respectively, all in electrical engineering.

He joined the Department of Electrical and Computer Engineering, Boston University, Boston, MA, as an Assistant Professor in 1992, where he is currently a Professor of electrical and computer engineering, biomedical engineering, and physics, and an Associate Director in the Center for Nanoscience and Nanobiotechnology. His current research interests include photonic materials, semiconductor optoelectronic devices, high-resolution microscopy and spectroscopy of semiconductor and biological materials, and biological sensing and imaging.

Dr. Ünlü was Chair of the IEEE Lasers and Electro-Optics Society, Boston Chapter from 1994 to 1995, winning the LEOS Chapter-of-the-Year Award. He was awarded the National Science Foundation Research Initiation Award in 1993, United Nations TOKTEN award in 1995 and 1996, and both the National Science Foundation CAREER and Office of Naval Research Young Investigator Awards in 1996. He was the former Chair of the IEEE LEOS Technical Committee on Photodetectors and Imaging, and currently, he is the Chair of the IEEE LEOS Nanophotonics Committee. He is an Associate Editor of the IEEE JOURNAL OF QuANTUM EleCtronics and Vice President of IEEE LEOS for Membership and Regional Activities-Americas.

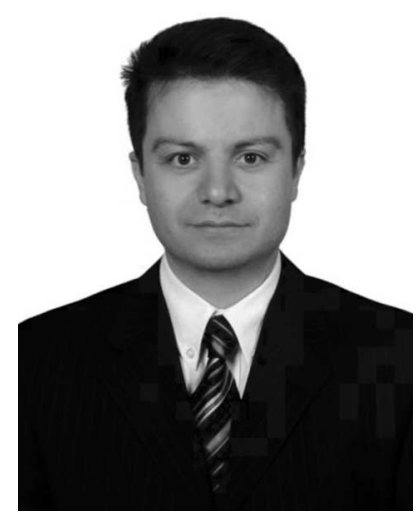

Ekmel Ozbay (M'98) was born on March 25, 1966, in Ankara, Turkey. He received the B.S. degree from Middle East Technical University, Ankara, Turkey, in 1983, and the M.S. and Ph.D. degrees from Stanford University, Stanford, CA, in 1989 and 1992, respectively, all in electrical engineering.

From 1992 to 1994, he was a Scientist in the DOE Ames National Laboratory at Iowa State University, Ames, working in the area of photonic bandgap materials. He joined Bilkent University, Ankara, Turkey, in 1995, where he is currently a Full Professor in the Department of Electrical and Electronics Engineering and the Department of Physics. His current research interests include metamaterials, GaN-based devices, photonic crystals, and high-speed optoelectronics. He has authored or coauthored more than 250 articles in various scientific journals, conference proceedings, and books.

Prof. Ozbay was the recipient of the 1997 Adolph Lomb Medal of the Optical Society of America and the 2005 European Union Descartes Science Award. Since 2002, he has been a

Topical Editor of Optics Letters.

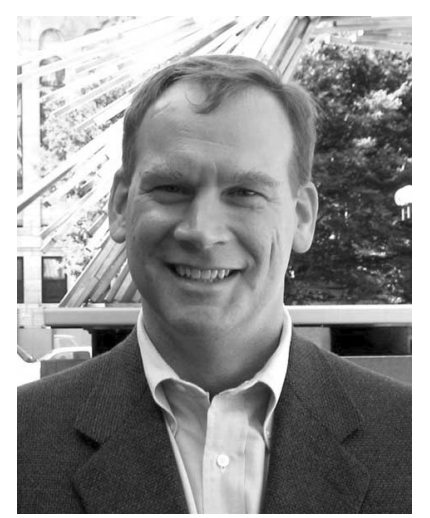

Bennett B. Goldberg (M'05) was born in Boston, MA, in 1959. He received the B.A. degree from Harvard College, Cambridge, MA, in 1982, and the M.S. and Ph.D. degrees in physics from Brown University, Providence, RI, in 1984 and 1987, respectively.

He was a Bantrell Postdoctoral Fellow at Massachusetts Institute of Technology and Francis Bitter National Magnet Laboratory in 1998. He is currently a Professor of physics, electrical and computer engineering, and biomedical engineering with Boston University, Boston, MA, where he is the Chairman of the Physics Department. He is the Director of Boston University's new Center for Nanoscience and Nanobiotechnology, an interdisciplinary center that brings together academic and industrial scientists and engineers in the development of nanotechnology with applications in materials and biomedicine. He has carried on research in near-field imaging of photonic bandgap, ring microcavity, and single-mode waveguide devices, and has recently developed subsurface solid immersion microscopy for Si inspection. His group is working on novel approaches to subcellular imaging with interferometric fluorescenent techniques, and in biosensor fabrication and development of waveguide evanescent bioimaging techniques. Nano-optics research includes Raman scattering of individual nanotubes and nano-optics of electron systems in quantum wells and quantum-dot structures. His current research interests incude the general area of ultrahigh-resolution microscopy and spectroscopy techniques for hard and soft materials systems. 


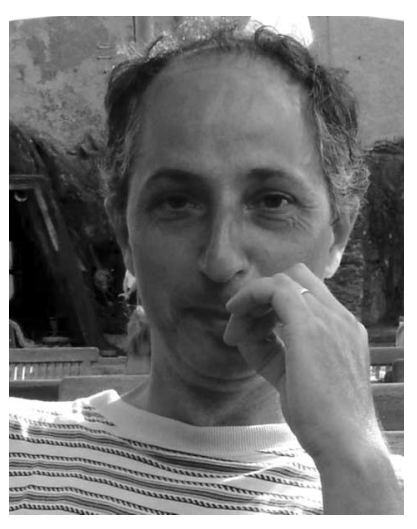

Niek F. van Hulst received the Ph.D. degree in molecular and laser physics from the University of Nijmegen, Nijmegen, The Netherlands, in 1986.

After research in nonlinear optics, organic materials, integrated optics, and waveguides, he became an Assistant Professor at the University of Twente, Enschede, The Netherlands, in 1990, and started research in near-field optical and atomic force microscopy, nonlinear optics, and hyper Raleigh scattering. In 1997, he became a Full Professor in applied optics at the MESA ${ }^{+}$Institute for Nanotechnology, Department of Science and Technology, University of Twente, focusing on single-molecule detection, nanophotonics, photonic structures, scanning probe technology, and applications in molecular biology and chemistry. Since September 2005, he has been an ICREA Research Professor and a Senior Group Leader in Nanophotonics at the Institute of Photonic Sciences (ICFO), Barcelona, Spain.

Prof. van Hulst received the European Science Award of the Körber Foundation in 2003, for his work on single-molecule detection. 